\title{
Sinonasal Adenocarcinoma, Salivary-Type
}

National Cancer Institute

\section{Source}

National Cancer Institute. Sinonasal Adenocarcinoma, Salivary-Type. NCI Thesaurus. Code C161007.

Adenocarcinomas that arise from the seromucinous glands and surface epithelium of the nasal cavity and paranasal sinuses. These adenocarcinomas morphologically resemble the adenocarcinomas that arise from the major and minor salivary glands. 